\title{
Comparação entre os resultados do teste da orelhinha e da timpanometria: devemos revisar o protocolo de triagem auditiva neonatal?
}

\section{Comparison of the results of the otoacoustic emission tests and tympanometry tests: should we revise the neonatal hearing screening protocol?}

Armanda de Oliveira Pache de Faria ${ }^{1}$, Alan Araújo Vieira ${ }^{1}$, Raphaella Costa Moreira Simen ${ }^{1}$ e Maria Elisa Vieira da Cunha Ramos Miterhof $^{1}$

'Universidade Federal Fluminense

\section{Palavras-chave:}

Triagem neonatal; Testes de impedância acústica; Orelha média; Recém-nascido.

\section{Resumo}

Introdução: a grande incidência de alterações na orelha média em recém-nascidos gera aumento na frequência de resultados falsamente alterados no teste da orelhinha. Objetivo: analisar a correlação entre as respostas das emissões otoacústicas evocadas transientes com os resultados da timpanometria. Métodos: estudo prospectivo no qual foram estudados os recém-nascidos que permaneceram internados na unidade neonatal. Estes foram submetidos à timpanometria com tom de $226 \mathrm{~Hz}$ e $1.000 \mathrm{~Hz}$ e a emissões otoacústicas evocadas transientes. As curvas timpanométricas foram classificadas em normais (A e duplo pico) ou alteradas (Ad, As, B, C). A população foi descrita por meio de frequências absolutas e relativas, e a correlação entre os resultados dos exames realizados foi estabelecida por meio do teste qui-quadrado e por regressão logística, com significância de 95\%, utilizando-se o pacote estatístico SPSS 16.0. Resultados: dos 172 pacientes admitidos, preencheram os critérios de inclusão 137, perfazendo 274 orelhas. Dessas, 22 apresentaram emissões otoacústicas evocadas transientes alteradas, 14 timpanometrias com tom de $226 \mathrm{~Hz}$ e 18 timpanometrias com tom de $1.000 \mathrm{~Hz}$. A correlação entre emissões otoacústicas evocadas transientes alteradas e timpanometria com tom de $226 \mathrm{~Hz}$ alterada no primeiro exame apresentou OR = 44 (IC 95\%: 11,75 - 166,81; p < 0,001); com a timpanometria tom de $1.000 \mathrm{~Hz}$, a correlação apresentou OR = 829 (IC 95\%: 100,2 - 6.879,17; p $<0,001)$. Conclusões: houve melhor correlação entre os resultados das emissões otoacústicas evocadas transientes com a timpanometria com tom de $1.000 \mathrm{~Hz}$ na população estudada.

\section{Keywords:}

Neonatal screening; Acoustic impedance tests; Ear, middle; Infant, newborn.

\footnotetext{
Abstract

Introduction: the high incidence of middle ear alterations in newborns causes increased frequency of falsely altered results in otoacoustic emission tests. Objective: to correlate the responses of transient evoked otoacoustic emissions with the results obtained with $226 \mathrm{~Hz}$ and $1,000 \mathrm{~Hz}$ tympanometry in newborns admitted to the Neonatal Unit. Methods: prospective study on newborns in the neonatal unit who were submitted to $226 \mathrm{~Hz}$ and $1,000 \mathrm{~Hz}$ tympanometry and transient evoked otoacoustic emissions. Tympanometric curves were classified as normal (A and Double Peak) or changed (Ad, As, B, C). The sample was described by absolute and relative frequencies, and the correlation between the results of the examinations was established by chi-square test and logistic regression, considering the significance of 95\%, using the statistical package SPSS 16.0. Results: 137 of the 172 infants admitted to the neonatal unit met the inclusion criteria, totaling 274 ears. Of this, 22 had transient evoked otoacoustic emissions changed, 14 tympanometry with $226 \mathrm{~Hz}$ probe and 18 with $1,000 \mathrm{~Hz}$ probe. The correlation between both altered transient evoked otoacoustic emissions and $226 \mathrm{~Hz}$ tympanometry in the first examination showed $\mathrm{OR}=44$ (95\%: 11.75 - 166.81, p < 0.001); with 1,000Hz tympanometry, the correlation presented OR $=829$ (Cl 95\%: 100.2 - 6,879.17; $\mathrm{p}<$ $0.001)$. Conclusions: there was a better correlation between the results of otoacoustic emissions with $1,000 \mathrm{~Hz}$ tympanometry in the population studied.
} 


\section{INTRODUÇÃO}

A audição constitui uma das principais habilidades do ser humano, sendo um dos importantes meios de ligação deste com o ambiente. Quanto mais cedo for identificada e abordada a deficiência auditiva, maiores as chances de a criança apresentar um desenvolvimento dentro dos padrões esperados para uma criança com audição normal. ${ }^{1,2,3}$

No Brasil, os dados são escassos, mas estima-se que em recém-nascidos (RN) de baixo risco a deficiência auditiva bilateral seja em média de 1 a 3 neonatos a cada 1.000 nascidos vivos, o que aumenta para algo em torno de 1 a 6 a cada 100 quando se trata de neonatos provenientes de unidade de terapia intensiva (UTI) neonatal. ${ }^{4}$

Os programas de triagem auditiva neonatal universal (Tanu) recomendam, atualmente, o uso das emissões otoacústicas evocadas (EOA) e o potencial evocado auditivo de tronco encefálico (Peate/Bera) para avaliar a possibilidade de deficiência auditiva em RN, com o intuito de diagnosticá-los e encaminhá-los precocemente para uma intervenção adequada e eficaz. No entanto, o resultado desses dois exames pode ser influenciado pela presença de afecções de orelha média, que gera falsos alterados, podendo, então, levar a um aumento da quantidade de RN que necessitem de reavaliação e acompanhamento audiológico, gastos hospitalares excessivos e estresse familiar. ${ }^{5-11}$

A timpanometria é um teste auditivo feito com o propósito de avaliar as condições da orelha média. Pode ser realizado com o tom sonda de $226 \mathrm{~Hz}$ e os resultados são confiáveis para o diagnóstico de alterações de orelha média em adultos e lactentes a partir de 6 meses de idade, no entanto a literatura questiona sua sensibilidade quando

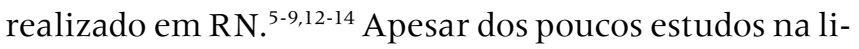
teratura abordando a timpanometria no neonato, o uso do tom sonda de $1.000 \mathrm{~Hz}$ (alta frequência), parece ser o mais adequado para a avaliação dessa população. ${ }^{5-9,12-15}$

O objetivo deste estudo é correlacionar os resultados das emissões otoacústicas evocadas transientes (EOATE) com as medidas da timpanometria com tom sonda de baixa $(226 \mathrm{~Hz})$ e de alta frequência $(1.000 \mathrm{~Hz})$.

\section{MÉTODO}

Estudo prospectivo, no qual foram avaliados todos os $\mathrm{RN}$ que permaneceram internados na unidade neonatal no período de junho de 2015 até março de 2016.

Foram excluídos aqueles que apresentavam síndromes genéticas associadas a alterações auditivas, crianças com história familiar de perda auditiva e alterações neurológicas, infecções congênitas e os óbitos (antes da realização do exame). Esses $\mathrm{R} N$ foram excluídos para priorizar, na amostragem, fatores que pudessem interferir com alterações da orelha média.

Os dados de cada RN foram coletados em fichas próprias, desde o nascimento até a alta hospitalar. Os dados maternos foram coletados de forma retrospectiva através do prontuário médico e, quando necessário, confirmados diretamente com a mesma durante sua internação.

Os neonatos realizaram triagem auditiva neonatal por EOATE. Todos os testes foram realizados com o neonato no berço ou no colo da mãe ou responsável, em sono natural ou estado tranquilo, sem agitação. A timpanometria foi o último exame realizado, pois a pressurização emitida através da sonda poderia despertá-lo.

Para a realização da triagem auditiva por meio de EOA por estímulo transiente, foi utilizado o equipamento portátil OtoRead SCR da marca Interacoustics, calibrado de acordo com as instruções do fabricante. Foi posicionada uma oliva no meato acústico externo do neonato, adequada ao tamanho deste e, posteriormente, foram utilizados os parâmetros 'passa/falha' descritos no protocolo do equipamento com estímulos tipo click, na intensidade de $83 \mathrm{~dB}$ nível de pressão sonora (NPS) e seis bandas de frequências testadas $(1.5,2,2.5,3,3.5$ e 4 $\mathrm{kHz}$ ). Foi considerado como critério 'passa' as respostas que se apresentavam numa relação sinal/ruído de $4 \mathrm{~dB}$ em pelo menos três bandas de frequência.

A timpanometria foi realizada com tons de sonda nas frequências de $226 \mathrm{~Hz}$ e $1.000 \mathrm{~Hz}$, com o analisador de orelha média Titan da marca Interacoustics, equipamento automático e portátil, calibrado de acordo com as instruções do fabricante. Foi introduzida uma oliva no meato acústico externo do neonato, adaptada ao tamanho deste, sendo a timpanometria realizada com a faixa de pressão variando entre $+300 \mathrm{daPa}$ a $-600 \mathrm{daPa}$, com velocidade de $50 \mathrm{daPa}$ /segundo e intensidade do tom teste de $85 \mathrm{~dB}$ NPS. Os neonatos foram avaliados primeiro com o tom sonda de $226 \mathrm{~Hz}$ e em seguida com o de $1.000 \mathrm{~Hz}$. A orelha na qual foi iniciado o teste não foi pré-definida, sendo a escolha dependente da posição do RN no colo da mãe ou no berço. Quando a curva timpanométrica não era obtida de forma satisfatória por movimentação do neonato ou por aparecer traçado não usual, o teste era repetido com a retirada da oliva e sua reinserção na mesma orelha para obtenção de nova curva timpanométrica.

Os timpanogramas foram classificados segundo Jerger (1970), ${ }^{16}$ Kei et al. $(2003)^{17}$ e Margolis et al. $(2003)^{15} \mathrm{em}$ :

a) curva tipo A - com pico único de admitância entre -150 e +100 daPa;

b) curva tipo Ad - pico de máxima complacência com amplitude aumentada; 
c) curva tipo Ar - pico de máxima complacência com amplitude reduzida;

d) curva tipo B - curva plana, sem pico de admitância;

e) curva tipo $\mathrm{C}$ - pico de admitância deslocado para pressão negativa;

f) curva tipo Dp - curva em duplo pico, considerada normal nos RN.

Para análise dos resultados, utilizou-se um critério simplificado de classificação dos timpanogramas: normais ou alterados. Foram considerados normais todos os timpanogramas do tipo A e duplo pico, e alterados os demais.

Os RN que tiveram algum exame alterado na primeira avaliação foram submetidos a uma segunda avaliação, em média, 30 dias após o primeiro exame. Os neonatos que permaneceram com os resultados dos exames alterados nessa segunda avaliação foram encaminhados para o ambulatório de otorrinolaringologia, dando continuidade à investigação.

Foram avaliadas as seguintes variáveis maternas, relacionadas à gestação e ao parto: idade materna, escolaridade, cor, número de consultas de pré-natal, presença de doenças hipertensivas, ${ }^{18}$ pressão arterial controlada, diabetes tipo 1 ou gestacional (diagnosticada durante a gravidez), aleitamento materno, tipo de parto, presença de corioamnionite, infecção de trato urinário e rotura prematura de membranas ovulares.

Os dados neonatais avaliados foram: peso de nascimento (em gramas), idade gestacional (em semanas; sendo levados em consideração, primeiramente, a data da última menstruação ou a ultrassonografia com até 12 semanas de gestação ou o método de Ballard), ${ }^{19}$ sexo, idade do $\mathrm{RN}$ em dias, sofrimento fetal, asfixia (Apgar $<7$ no $5^{\circ}$ minuto de vida), dias de uso de fototerapia, uso de aminoglicosídeo, suporte ventilatório e hipoglicemia.

As variáveis contínuas foram descritas pelas medidas de tendência central e dispersão (média, mediana, desvio-padrão, valor mínimo e máximo), e as variáveis categóricas foram descritas por meio da frequência absoluta e relativa.

A correlação entre as medidas de EOATE e timpanometria com tom sonda de $226 \mathrm{~Hz}$ e $1.000 \mathrm{~Hz}$ foram realizadas por meio do teste qui-quadrado e quantificadas por regressão binária logística com nível de significância de $95 \%$, utilizando o pacote estatístico SPSS 16.0 for Windows. O presente estudo foi aprovado pelo Comitê de Ética em Pesquisa sob o Certificado de Apresentação para Apreciação Ética n. 44050915.3.0000.5243.

\section{RESULTADOS}

Foram admitidos na unidade neonatal $172 \mathrm{RN}$. Destes, 35 foram excluídos (14 óbitos, 9 RN com síndromes genéticas associadas a alterações auditivas, $7 \mathrm{RN}$ com infecções congênitas, $4 \mathrm{RN}$ transferidos e $1 \mathrm{RN}$ com meningite).

Foram avaliados $137 \mathrm{RN}$ (274 orelhas), sendo 74 do sexo feminino $(54,0 \%)$ e 63 do sexo masculino $(46,0 \%)$, com idade média de 5 dias na realização do exame e idade gestacional entre 24 e 42 semanas, com média de 36,35 . O peso variou entre 735 gramas e 4.105 gramas, com média de $2.658,28$. A quantidade mínima de consultas no pré-natal foi de duas (média de 7,70 e mediana de 8$)$.

A idade média materna foi de 27,30 e a maioria era não branca (67,4\%). Quanto à escolaridade, 51,4\% das mulheres tinham nível médio completo.

Cerca de 57,9\% das mulheres apresentavam alguma doença hipertensiva, sendo que $74,6 \%$ tiveram a pressão arterial controlada durante a gestação. A metildopa foi o medicamento anti-hipertensivo mais utilizado $(33,3 \%)$.

A frequência de diabetes tipo $1(4,3 \%)$ e gestacional $(8,0 \%)$ foi baixa na população estudada. O tipo de parto mais frequente foi o cesáreo $(74,6 \%)$ e o aleitamento materno esteve presente em $119(86,2 \%)$ das parturientes. O risco infeccioso esteve presente em $36,3 \%$ das gestantes (infecção periparto de trato urinário, rotura prematura de membranas ovulares e corioamnionite). Pelo menos $43 \%$ da população de RN foi submetida a algum suporte ventilatório; 18 foram intubados e acoplados em ventilação mecânica e 39 fizeram uso de CPAP nasal. Do total de RN, $40 \%$ eram prematuros, $38 \%$ tinham peso menor que 2.500 gramas e $12,3 \%$ tinham peso menor que 1.500 gramas (Tabela 1 ).

Na primeira avaliação realizada nos $\mathrm{RN}$ estudados, foram encontradas EOATE alteradas em $16 \%$ dos resultados. Na timpanometria com tom sonda de $226 \mathrm{~Hz}$ e com tom sonda de $1.000 \mathrm{~Hz}$ foram encontrados, respectivamente, $10,1 \%$ e $13,0 \%$ de exames alterados.

Na segunda avaliação, 3,6\% das EOATE permaneceram alteradas. A timpanometria com tom sonda de 226 Hz não apresentou resultados alterados, ao contrário da timpanometria com tom sonda de $1.000 \mathrm{~Hz}$, que permaneceu com três $(2,2 \%)$ exames alterados.

Ter sido prematuro não aumentou a probabilidade de apresentar EOATE e timpanometria com tom sonda de $226 \mathrm{~Hz}$ e de $1.000 \mathrm{~Hz}$ alterados nos RN estudados. Quando o grupo de RN estudados foi dividido em relação ao peso de nascimento menor ou maior que 1.500 gramas, a timpanometria com tom sonda de $1.000 \mathrm{~Hz}$ 
Tabela 1 - Distribuição de frequência das variáveis estudadas

\begin{tabular}{|c|c|c|c|}
\hline VARIÁVEIS MATERNAS & & FREQUÊNCIA & $\%$ \\
\hline Escolaridade & Médio completo & 71 & 51,4 \\
\hline Cor & Não branca & 93 & 67,4 \\
\hline Parto cesáreo & & 103 & 74,6 \\
\hline Doenças hipertensivas & & 80 & 57,9 \\
\hline Pressão arterial controlada & & 103 & 74,6 \\
\hline \multirow{2}{*}{ Diabetes } & Tipo 1 & 6 & 4,3 \\
\hline & Gestacional & 11 & 8,0 \\
\hline Aleitamento materno & & 119 & 86,2 \\
\hline Risco infeccioso & & 50 & 36,3 \\
\hline VARIÁVEIS RN & & FREQUÊNCIA & $\%$ \\
\hline Uso de aminoglicosídeo & & 34 & 24,6 \\
\hline Sofrimento fetal & & 34 & 24,6 \\
\hline Uso de fototerapia & & 26 & 18,8 \\
\hline Suporte ventilatório & & 60 & 43,4 \\
\hline Sexo masculino & & 63 & 46,0 \\
\hline Asfixia & & 26 & 18,8 \\
\hline Hipoglicemia & & 19 & 13,8 \\
\hline Prematuridade & & 55 & 39,9 \\
\hline Peso $<1.500 \mathrm{~g}$ & & 17 & 12,3 \\
\hline
\end{tabular}

apresentou o mesmo percentual de alteração que a EOATE $(20,6 \%)$, ou seja, os mesmos RN apresentaram testes alterados (Tabela 2).

Houve correlação dos resultados alterados da timpanometria com tom sonda de $226 \mathrm{~Hz}$ e de $1.000 \mathrm{~Hz}$ com a presença de alterações nas EOATE, tanto na primeira quanto na segunda avaliação. Na primeira avaliação, a correlação entre emissão otoacústica e timpanometria com tom sonda de $226 \mathrm{~Hz}$ alterada, apresentou OR de 44,27 (IC 95\%: 11,75 - 166,81; p < 0,001). No entanto, a correlação entre emissão otoacústica e timpanometria com tom sonda de $1.000 \mathrm{~Hz}$, apresentou OR de 829,50 (IC 95\%:100,02-6.879,17; p <0,001). Na segunda avaliação não houve correlação entre as medidas de emissão otoacústica e timpanometria com tom sonda de $226 \mathrm{~Hz}$. No entanto, a timpanometria com tom sonda de 1.000 $\mathrm{Hz}$ ainda apresentou boa correlação (OR 33; IC 95\%: $1,74-627,28 ; p=0,02$ ) (Tabela 3).

\section{DISCUSSÃO}

Atualmente, na literatura médica, tem sido discutido o manejo do RN quando a triagem auditiva neonatal detecta alguma alteração em seus resultados. A possibilidade de alterações transitórias alterarem os resultados e a necessidade de dar seguimento à investigação, quando isto ocorre, faz com que vários autores sugiram a inclusão de procedimentos que auxiliem no diagnóstico diferencial entre as perdas auditivas. ${ }^{5-7,9,12}$ A timpanometria ajuda a fornecer informações sobre a orelha média e, ao se diagnosticar estas alterações, o direcionamento ao diagnóstico de certeza nesses $\mathrm{RN}$ deve ser reavaliado. ${ }^{20}$

Nesse estudo, foi observado um maior número de alterações de orelha média na primeira avaliação dos RN, representada pelo grande percentual de timpanogramas com tom sonda de $226 \mathrm{~Hz}$ e tom sonda de $1.000 \mathrm{~Hz}$ alterados, e que não se perpetuou na segunda avaliação. Esses achados corroboram os de um estudo realizado por Garcia e colaboradores, no qual os autores observaram que a prevalência de alterações em uma população com indicadores de risco para perda auditiva foi de 1,6/100 neonatos com perdas auditivas neurossensoriais e de 2,9/100 neonatos com perdas auditivas condutivas (alterações de orelha média e/ou cadeia ossicular). ${ }^{21}$

Importante ressaltar que RN com alterações auditivas atribuídas a disfunções na orelha média, como as otites secretoras, teriam maior risco para desenvolver otite média crônica, merecendo, portanto, acompanhamento diferenciado no primeiro ano de vida. ${ }^{14,22,23}$ Este fato enfatiza a necessidade de um diagnóstico diferencial bem-feito e justifica a inclusão da timpanometria na Tanu. A dúvida permanece em relação ao melhor tom sonda a ser utilizado em RN.

Neste estudo, a correlação entre EOATE e timpanometria com tom sonda de $226 \mathrm{~Hz}$ e tom sonda de $1.000 \mathrm{~Hz}$ foi avaliada por regressão binária logística, quantificando-as, o que difere dos outros estudos até o 
Tabela 2 - Comparação da EOATE e da timpanometria com tom sonda de $226 \mathrm{~Hz}$ e de $1.000 \mathrm{~Hz}$ na população estudada quanto à presença de prematuridade

\begin{tabular}{|c|c|c|c|}
\hline & $\begin{array}{c}\text { Prematuro (<37 sem.) } \\
\mathrm{n}(\%)\end{array}$ & $\begin{array}{c}\text { A termo } \\
\mathrm{n}(\%)\end{array}$ & p valor \\
\hline \multicolumn{4}{|l|}{$\overline{1^{a} \text { AVALIAÇÃO }}$} \\
\hline EOATE alterada & $9(16,4)$ & $13(15,7)$ & 0,546 \\
\hline Timpanometria $226 \mathrm{~Hz}$ alterada & $4(7,3)$ & $10(12)$ & 0,271 \\
\hline Timpanometria $1.000 \mathrm{~Hz}$ alterada & $8(14,5)$ & $10(12)$ & 0,428 \\
\hline \multicolumn{4}{|l|}{ 2ª AVALIAÇÃO } \\
\hline EOATE alterada & $2(3,6)$ & $3(3,6)$ & 0,664 \\
\hline Timpanometria $226 \mathrm{~Hz}$ alterada & 0 & 0 & - \\
\hline \multirow[t]{2}{*}{ Timpanometria $1.000 \mathrm{~Hz}$ alterada } & $1(1,8)$ & $2(2,4)$ & 0,651 \\
\hline & $\begin{array}{c}<1.500 \mathrm{~g} \\
\mathrm{n}(\%)\end{array}$ & $\begin{array}{c}>1.500 \mathrm{~g} \\
\mathrm{n}(\%)\end{array}$ & p valor \\
\hline \multicolumn{4}{|l|}{ 1a AVALIAÇÃO } \\
\hline EOATE alterada & $7(20,6)$ & $29(12,0)$ & 0,132 \\
\hline Timpanometria $226 \mathrm{~Hz}$ alterada & $3(8,8)$ & $13(5,4)$ & 0,317 \\
\hline Timpanometria $1.000 \mathrm{~Hz}$ alterada & $7(20,6)$ & $22(9,2)$ & 0,050 \\
\hline \multicolumn{4}{|l|}{ 2a AVALIAÇÃO } \\
\hline EOATE alterada & 0 & $9(3,7)$ & 0,301 \\
\hline Timpanometria $226 \mathrm{~Hz}$ alterada & 0 & 0 & - \\
\hline Timpanometria $1.000 \mathrm{~Hz}$ alterada & 0 & $4(1,7)$ & 0,064 \\
\hline
\end{tabular}

EOATE - emissões otoacústicas evocadas transientes.

Tabela 3 - Avaliação da correlação entre a timpanometria de 226 Hz e 1.000 Hz com as EOATE (regressão logística binária)

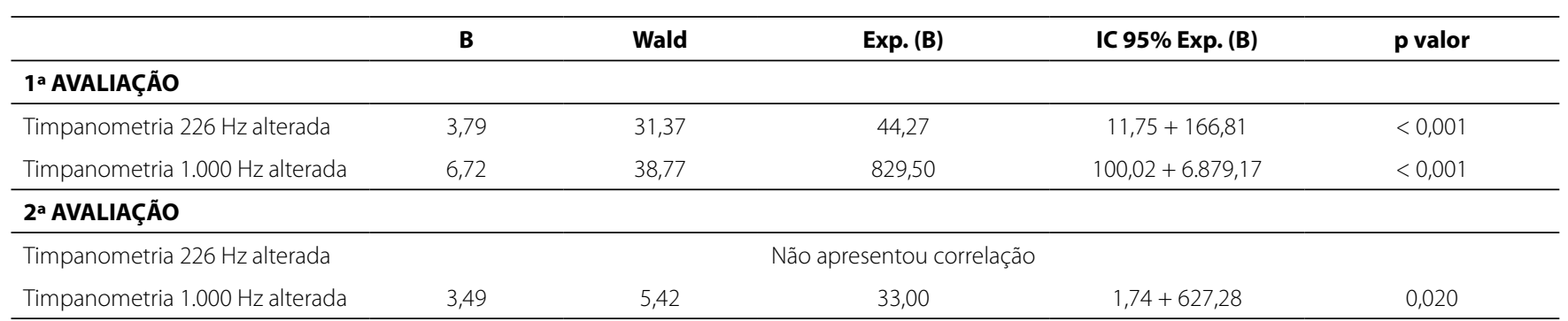

momento. ${ }^{20,24,25}$ A quantificação da correlação entre esses exames dá uma real ideia da importância de se escolher o tom sonda $1.000 \mathrm{~Hz}$, como já foi sugerido em outros estudos $7,8,10,12,16,20,25$ em detrimento do tom sonda $226 \mathrm{~Hz}$ (Tabela 3). No entanto, a correlação com o tom sonda $226 \mathrm{~Hz}$ também foi significativa.

A população avaliada no presente estudo apresentou grande percentual de afecções de orelha média na primeira avaliação. Este fato pode ser explicado pelos critérios de seleção empregados no estudo, direcionados para uma população de RN com alto risco para tais afecções.

Outro achado também interessante foi que a incidência de EOATE e timpanometrias alteradas não foi maior nos RN prematuros, quando comparados aos RN a termo. Provavelmente, este fato se deve à realização dos exames nos RN prematuros em um período mais tardio, pois estes se encontravam mais instáveis clinicamente e tiveram mais tempo para adaptar suas orelhas ao meio externo. Ao contrário, os RN a termo que necessitaram da UTI, porém, não permaneceram instáveis por tanto tempo, tiveram os exames da Tanu realizados com menor tempo de vida (entre 24-48h), o que, consequentemente, poderia gerar mais alterações condutivas por alterações transitórias de orelha média.

Foram relatados por Margolis et al. ${ }^{8}$ resultados semelhantes, nos quais observa-se que a quantidade de neonatos prematuros que não apresentou alteração no resultado dos timpanogramas com tom sonda de $1.000 \mathrm{~Hz}$ foi maior quando comparado à quantidade de RN a termo, sugerindo que a idade média com que o exame foi realizado pode ter contribuído para tal resultado, assim como no presente estudo.

Quando o grupo de RN estudados foi dividido em relação ao peso de nascimento menor ou maior que 1.500 gramas, a timpanometria com tom sonda de $1.000 \mathrm{~Hz}$ apresentou excelente correlação com o resultado das 
EOATE. Dos sete RN com EOATE ausentes, a timpanometria com tom sonda de $1.000 \mathrm{~Hz}$ detectou alteração em orelha média nos sete neonatos. O mesmo não ocorreu com o uso do tom sonda de $226 \mathrm{~Hz}$ : apenas três correspondências com as EOATE alteradas. Esses achados são compatíveis com outros trabalhos relatados na literatura. ${ }^{6,8,9,20,22}$

Baldwin ${ }^{26}$ apresenta, em seu estudo, timpanogramas normais apesar da presença de alteração em orelha média, quando o tom teste de $226 \mathrm{~Hz}$ foi utilizado. Rhodes e colaboradore ${ }^{27}$ evidenciaram uma alta taxa de falso-positivos quando correlacionaram os achados da timpanometria com tom sonda de $226 \mathrm{~Hz}$ aos achados do Peate/Bera e da EOA.

Além da maior frequência de alterações de orelha média nessa população de risco e maior necessidade de procedimentos que podem aumentar a incidência dessas alterações (tais como o uso de suporte ventilatório, a presença de sonda em cavidade oral e nasal, entre outros), é possível citar também as alterações anatômicas da orelha externa e média (menor tamanho do conduto auditivo, maior porção cartilaginosa em relação à óssea, o que torna o conduto auditivo do RN mais flácido) como fatores que irão influenciar os resultados dos timpanogramas, caso o tom sonda da timpanometria não seja adequadamente selecionado. Os componentes de massa são maiores com o tom sonda de $1.000 \mathrm{~Hz}$, sendo o mais recomendado para avaliar a orelha média do RN e do lactente jovem, no qual há uma maior contribuição da massa para a impedância da orelha média. ${ }^{11,15,26,28}$

Cabe levar em consideração as limitações metodológicas deste estudo, entre elas a amostragem por conveniência, ou seja, foram avaliados apenas $\mathrm{RN}$ que estiveram na UTI e na unidade intermediária neonatal e que os pais concordaram em participar do estudo, além de um rígido critério de exclusão com o intuito de priorizar RN com risco para alterações auditivas da orelha média. Também não houve cálculo de n amostral, limitando-se a inclusão de pacientes no estudo a um tempo pré-determinado (nove meses).

Neste estudo, também, não foi realizada a otoscopia para a confirmação de alterações da orelha média. A realização deste procedimento é indiscutivelmente necessária, porém não é a realidade na grande maioria dos serviços de triagem auditiva neonatal. Geralmente, a Tanu é realizada por fonoaudiólogos não capacitados para a realização de otoscopia; o único procedimento que é autorizado a realizar, nesses casos, é a meatoscopia, o que não confirmaria o diagnóstico de alterações em orelha média.

\section{CONCLUSÕES}

Na população estudada, a timpanometria com tom sonda de $1.000 \mathrm{~Hz}$ apresentou maior correlação com os resultados das EOATE do que o tom sonda de 226 $\mathrm{Hz}$; as alterações em orelha média são frequentes em $\mathrm{RN}$ internados em unidade neonatal e, em boa parte desses neonatos, essa alteração não se perpetuou numa segunda avaliação; a prematuridade não aumentou a chance de ter EOATE e timpanometria com tom sonda de $226 \mathrm{~Hz}$ e tom sonda de $1.000 \mathrm{~Hz}$ alterados; além disso, os RN de muito baixo peso (< 1.500 gramas) apresentaram boa correlação entre os resultados da EOATE e os da timpanometria com tom sonda de $1.000 \mathrm{~Hz}, \mathrm{o}$ que não ocorreu com a timpanometria com tom sonda de $226 \mathrm{~Hz}$. 


\section{REFERÊNCIAS}

1 Northern JL, Downs MP. Audição na infância. 5. ed. Rio de Janeiro: Guanabara Koogan; 2005.

2 Colella-Santos MF, Bragato GR, Martins PMF, Dias AB. Triagem auditiva em escolares de 5 a 10 anos. Rev CEFAC. 2009;11(4):644-53.

3 Yoshinaga-Itano C, Sedey AL, Coulter DK, Mehl AL. Language of early-and later-identified children with hearing loss. Pediatrics. 1998;102(5):1161-71.

4 Alvarenga KF, Piza MRT. Identificação e avaliação auditiva do recém-nascido e da criança. In: Caldas Neto S, Mello Júnior JF Martins RHG, Costa SS (Eds.). Tratado de otorrinolaringologia e cirurgia cervicofacial da ABORL-CCF. 2. ed. São Paulo: Editora Roca; 2011. v. I, p. 489-521.

5 Carmo MP, Costa NTO, Momensohn-Santos TM. Tympanometry in infants: a study of the sensitivity and specificity of $226-\mathrm{Hz}$ and 1,000-Hz probe tones. Int. Arch. Otorhinolaryngology. 2013;17(4):395-402.

6 Shahnaz N, Miranda T, Polka L. Multifrequency tympanometry in neonatal intensive care unit and well babies. J Am Acad Audiol. 2008;19(5):392-418

7 Hoffmann A, Deuster D, Rosslau K, Knief A, Am ZehnhoffDinnesen A, Schmidt CM. Feasibility of $1,000 \mathrm{~Hz}$ tympanometry in infants: tympanometric trace classification and choice of probe tone in relation to age. Int J Pediatr Otorhinolaryngol. 2013;77(7):1198-203.

8 Margolis RH, Bass-Ringdahl S, Hanks WD, Holte L, Zapala DA Tympanometry in newborn infants - 1kHz norms. J Am Acad Audiol. 2003;14(7):383-92.

9 Calandruccio L, Fitzgerald TS, Prieve BA. Normative multifrequency tympanometry in infants and toddlers. J Am Acad Audiol. 2006;17(7):470-80.

10 American Academy of Pediatrics, Joint Committee on Infant Hearing. Year 2007 position statement: principles and guidelines for early hearing detection and intervention programs. Pediatrics. 2007;120(4):898-921.

11 Lewis DR, Marone SA, Mendes BC, Cruz OL, Nóbrega Md. Multiprofessional committee on auditory health: COMUSA. Braz J Otorhinolaryngol. 2010;76(1):121-8.

12 Alaerts J, Luts H, Wouters J. Evaluation of middle ear function in young children: clinical guidelines for the use of 226- and 1,000-Hz tympanometry. Otol Neurotol. 2007;28(6):727-32.

13 Paradise JL, Smith CG, Bluestone CD. Tympanometric detection of middle ear effusion in infants and young children. Pediatrics. 1976;58(2):198-210.

14 Doyle KJ, Kong YY, Strobel K, Dallaire P, Ray RM. Neonatal middle ear effusion predicts chronic otitis media with effusion. Otol Neurotol. 2004;25(3):318-22.
15 Margolis RH, Hunter LL. Timpanometria: princípios e aplicações clínicas. In: Musiek FE, Rintelmann WI (Eds.). Perspectivas atuais em avaliação auditiva. São Paulo: Manole; 2001. p. 85-126.

16 Kei J, Allison-Levick J, Dockray J, Harrys R, Kirkegard C, Wong $\mathrm{J}$ et al. High-frequency $(1,000 \mathrm{~Hz})$ tympanometry in normal neonates. J Am Acad Audiol. 2003;14(1):20-8.

17 Jerger J. Clinical experience with impedance audiometry. Arch Otolaryngol. 1970;92(4):311-24.

18 Ministério da Saúde. Secretaria de Atenção à Saúde. Departamento de Ações Programáticas e Estratégicas. Gestação de alto risco: manual técnico. 5. ed. Brasília: Ministério da Saúde; 2012. (Série A. Normas e manuais técnicos).

19 Alexander GR, Himes JH, Kaufman RB, Mor J, Kogan M. A United States national reference for fetal growth. Obstet Gynecol. 1996;87(2):163-8.

20 Carmo MP, Almeida MG, Lewis DR. Timpanometria com tons teste de $226 \mathrm{~Hz}$ e $1 \mathrm{kHz}$ em um grupo de lactentes com indicadores de risco para deficiência auditiva. Rev Soc Bras Fonoaudiol. 2012;17(1):66-72.

21 Garcia CFD, Isaac ML, Oliveira JAA. Emissão otoacústica evocada transitória: instrumento para detecção precoce de alterações auditivas em recém-nascidos a termo e pré-termo. Rev Bras Otorrinolaringol. 2002;68(3):344-52.

22 Boone RT, Bower CM, Martin PF. Failed newborn hearing screens as presentation for otitis media with effusion in the newborn population. Int J Pediatr Otorhinolaryngol. 2005;69(3):393-7.

23 Engel J, Mahler E, Anteunis L, Marres E, Zielhuis G. Why are NICU infants at risk for chronic otitis media with effusion? Int J Pediatr Otorhinolaryngol. 2001;57(2):137-44.

24 Garcia MV, Azevedo MF, Testa JR. Accoustic immitance measures in infants with 226 and 1,000Hz probes: correlation with otoacoustic emissions and otoscopy examination. Braz J Otorhilaryngol. 2009;75(1):80-9.

25 Camboim ED, Correia AMN, Vasconcelos D, Torres R, Scharlach RC, Azevedo MF. Análise comparativa das emissões otoacústicas com a timpanometria em lactentes de 0 a 6 meses. Rev CEFAC. 2012;14(3):403-12.

26 Baldwin $M$. Choice of probe tone and classification of trace patterns in timpanometry undertaken in early infancy. Int J Audiol. 2006;45(7):417-27.

27 Rhodes MC, Margolis RH, Hirsch JE, Napp AP. Hearing screening in the newborn intensive care nursery: comparison of methods. Otolaryngol Head Neck Surg. 1999;120(6):799-808.

28 Lyra e Silva Kde A, Novaes Bde A, Lewis DR, Carvallo RM. Tympanometry in neonates with normal otoacoustic emissions: measurements and interpretation. Braz J Otorhilaryngol. 2007;73(5):633-9. 